\title{
Mini Windmill Generator Kit for Homework for Hiroshima Univ. Monozukuri Junior Doctor Special Educational Program
}

\author{
Hiroyuki Y. Suzuki, Kazuo Kawada, Masayasu Nagamatsu \\ Graduate School of Humanities and Social Sciences, Department of Educational Science, Hiroshima University, \\ 1-1-1-Kagamiyama, Higashi-Hiroshima City, Hiroshima 739-8524, Japan \\ E-mail:hiro-suzuki@hiroshima-u.ac.jp,kawada@hiroshima-u.ac.jp,nagamatu@hiroshima-u.ac.jp
}

\begin{abstract}
Hiroshima University Monozukuri Junior Doctor is a special educational program for young (11 - 15 in age) students. It had to be stopped, however, at the midst because of COVID-19 epidemic. We changed it to homework based one. Modified "Mini Windmill Generator Kit" were sent to them with a manual of "Procedure of quantitative research" for self-learning. The result shows that several specific tendencies when the data summarized statistically, although the data collected with different places with different conditions.
\end{abstract}

Keywords: Technology education, STEM, Quantitative evaluation, Moment of inertia

\section{Introduction}

Hiroshima University Monozukuri Junior Doctor is a special educational program for young (11-15 in age) volunteered students, aimed for cultivating innovative and outstanding young people as leaders for next generation. Original idea of the program is proposed by Japan Science and Technology Agency (JST).

Our program has started in Jul. 2019, giving 5 seminars in Pre-Stage to around 300 participants (5 times of originally recruited number), and selected to 40 for FirstStage by the end of Sept. The First-Stage also went on smoothly, giving three seminars to the end of 2019. However, following seminars had to be cancelled because of COVID-19 epidemic. We had to change all the following program to self-learning ones. This report presents details of first homework for First-Stage student.

\section{Characteristics of Our Program}

Our program is composed of three stages of Pre-Stage (recruit of 60 participants), First-Stage (selected to 40) and Second-Stage (selected to 20), in which there are selections at each stage-up timing. This selection policy is based on JST's requirement, that is, only few excellent students will be selected "individually" and continue to study throughout the program. Nonetheless, we introduce distinctive characteristics of "group activity" to the program (approved by JST). We believe that selection of only few "individual" leaders is somehow oversimplified vision to compete with today's science and technology developments. Many of current problems are resolved by, sometimes very large, projects.

We are going to find and cultivate young people of distinctive talents, not only the merely innovative leaders but also capable supporters of innovators, by the program. Proper mixing of diverse talents is a key of success for many kinds of today's projects.

\section{Monozukuri Seminar using Mini Windmill Generator Kit}

We originally introduced Mini Windmill Generator Kit (Artec co., Japan) in First-Stage's third seminar. The windmill generator has weathercock appearance, that is, it always turns to upwind direction automatically. Torque 
generated by the wind at the tail fin makes the body turn. Proper size of tail fin and the smoothly moved bearing under the body are the keys for smooth function.

Two themes were given to students (9 groups, 4-5 member each) .

- $\quad$ Finding proper size and shape of tail fin.

- Making smoothly moving bearing using 3D printer.

\subsection{Finding proper size and shape of tail fin}

Plastic sheet of $0.8 \mathrm{~mm}$ was used to make tail fins. The sheet was easy to be cut by scissors and set to the rear of the body with a screw (a screw hole was added). Wind was given by electric fans and generated voltages were measured by a tester changing "parameters" of power of electric fan, distance between fan and generator, etc.

We modified the kit to be able to measure the generated voltages by extending thin lead wires from the generator. Additionally, turning torques of the whole body (with fins) were measured using a jig we prepared (Fig. 1).

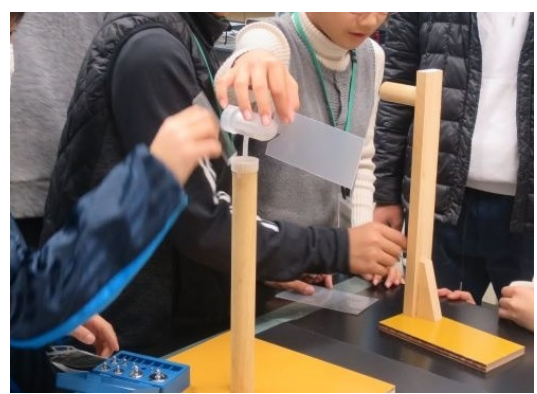

Fig. 1 Jig for measuring turning torque of the windmill generator body.

Generated torque by wind is in proportion to the size of the fin. At the same time, the required torque to turn the whole body is also in proportion to the moment of inertia. That is, the larger the fin is, the larger the torque is required to turn the body. They are trade-off relationship each other. The optimum shape and size of the tail fin will be found at the balanced point of those two factors.

Students were expected to find appropriate sizes and shapes of fins by themselves. We did not give, however, detailed lecture on above mentioned mechanism beforehand. We rather expected that students grabbed overall picture of the mechanisms by themselves through quantitative data collection and arrangement of the data using tables and graphs.

\subsection{Making smoothly moving bearing using 3D printer}

Students were experienced 3D printing as an introduction to digital fabrication. They firstly studied basic commands of 3D CAD (Design spark Mechanical) and "extrude" concentric shapes with a hole, namely bearing, in monitor. Then, they made the bearings data to reality, using 3D printer (XYZ printing PRO).

Majority of the students were successful to draw bearing design but only few were printed, because of limitation of printing speed. Therefore, theme 3.1 was performed using original bearings in the kit.

\subsection{Result of seminar}

Each team could take data of tail fins performance by changing distance between electric fan and the generator and power needed to turn the fins. Group work made easy to perform experiments in shorter time.

They were eager to propose new design of tail fins, to collect data by experiments, to design bearings in $3 \mathrm{D}$ $\mathrm{CAD}$ by themselves. Nonetheless, sufficient data were not collected for the discussion in the seminar, because of the time limitation (a half-day seminar). We were going to continue the same theme, changing approach rout, in following seminar.

\section{Homework}

\subsection{Preparation of homework kit}

All meetings were banned by Univ. official, after rising COVID-19 epidemic. In Mar. 2020, we sent a homework kit of mini windmill generator to every student, as supplemental contents of previous seminar.

Three color LEDs are added (with a series resistor) as voltage indicator. A tester is attached to the kit. Manual of "Procedure of quantitative research" in which we roughly explained how to figure the graphs and encouraged them to "find a peak value" in figures. Note that we did not designate any parameters to be changed. All the experimental conditions were decided by oneself. In homework, we gave two themes of,

- $\quad$ finding best design of tail fin (same as seminar)

- $\quad$ finding best design of windmill.

The windmills could easily made by cutting pet PETbottles. The kit contained a coupler to unite PET-bottle windmill to generator axle. 


\subsection{Reports returned from students}

An example of report returned from a student (female, first grade in junior high school) is shown in Fig. 2.

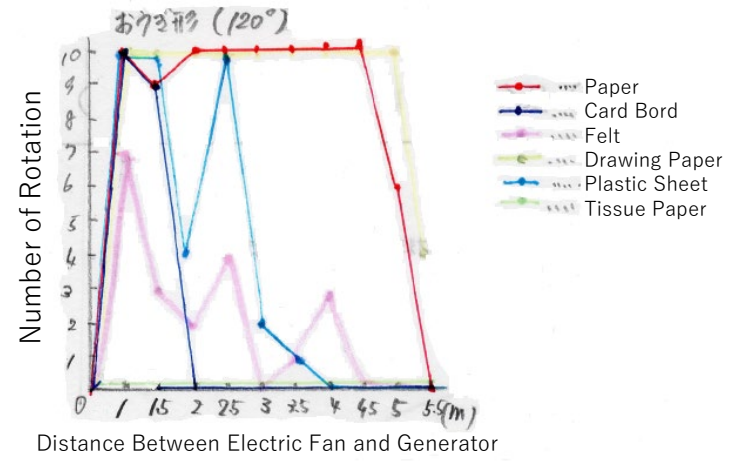

Fig. 2 Research for finding best material for tail fin.

She tried to evaluate the effect of materials on the performance of tail fin. She chose fan shape fins (radius of $100 \mathrm{~mm}$ and angle of 120 deg.) made of different materials of, i) copy paper, ii) card-bord, iii) felt, iv) drawing paper, v) plastic sheet and vi) tissue paper.

Number of rotation (reacted to the wind) were counted changing distance between electric fan and the generator. She concluded that the drawing paper showed the best performance. She also checked size of the fins in other part of the report and concluded about $100 \mathrm{~cm}^{2}$ was the optimum size.

Figure 3 is an example of report on windmill experiment from another student (female, first grade in junior high school), showing various windmills. She checked windmill performance from two viewpoints of, i) number of blades (upper table and figure) and ii) length of blade (lower table and figure) and summarized that the optimum number of blades was three and size of blade was $3 \mathrm{~cm}$, respectively.

Students made plan of experiment by themselves since we did not give step-by-step procedure in the instruction manual but gave basic idea of appropriated data collection, so that somehow unique trials were performed by many students.

\section{Discussions}

Windmill generators are one of popular teaching materials for energy conversion since it is easy to make windmills by hands. However, there are hurdles when we try to understand "the essence" of the product, since it

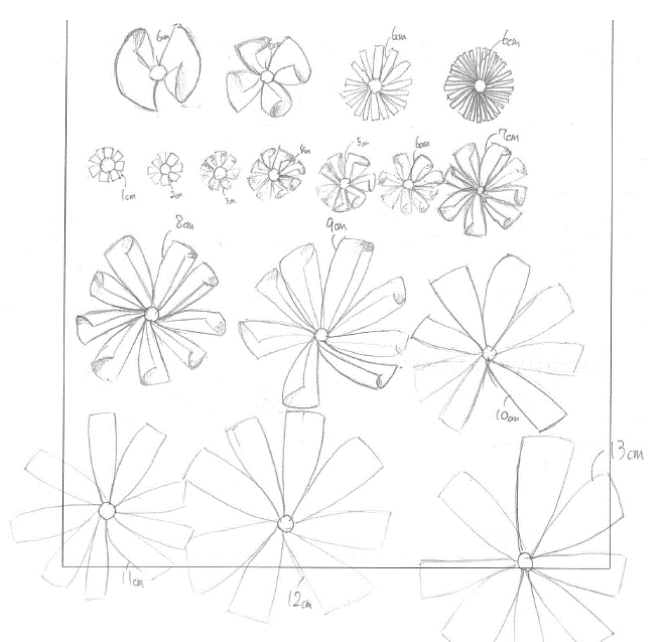

Fig. 3 Performance of many designs of PET-bottle windmills were measured by a student.

contains "complexity" as "real product". It contains all STEM factors. Therefore, some research gave up quantitative evaluations by students ${ }^{1)}$ or evaluated only by instructors ${ }^{2,3)}$. Some research came into quantitative evaluations, but they still scarcely used graphs ${ }^{4,5)}$.

Our program is forced to introduce, by chance, experiments planned and performed by students "individually". We introduced quantitative evaluation using graphs, but experimental parameters and conditions were all different in each student. Maximum voltages attained were varied from $0.3 \mathrm{~V}$ to $6 \mathrm{~V}$, for example, among their experiments. Nonetheless, there

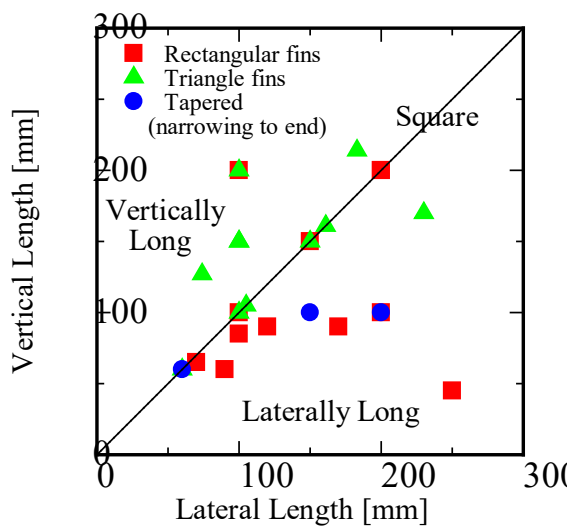

Fig. 4 Optimum sizes and shapes of tail fins found by each student experiment. 
are several noteworthy tendencies can be found when we summarize their data statistically.

Figure 4 shows the lateral and vertical sizes of tail fins of the best performance. Although argument for selecting the best fin were merely scattered, there is tendency between shape and aspect ratio of fins. Rectangular and tapered (narrowing to the end) fins are relatively laterally long. In contrast, triangle fins (widen to the end, including fan shape) have vertically long. Higher moment of inertia of triangular ones must be one reason of such difference. Interestingly, on the other hand, sizes of fins are almost indifferent to their shapes.

Materials of fins also have no considerable effect to sizes and shapes of them, as shown in Fig. 5, although the result in Fig. 2 by a student indicates clear difference. Much higher weight of generator body may mask the weight differences of fin materials.

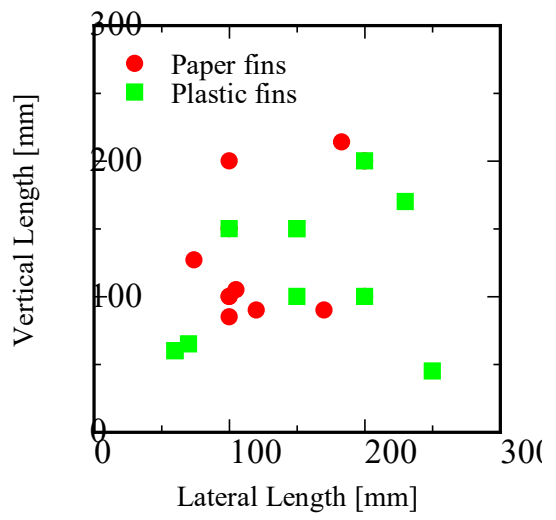

Fig. 5 Effect of materials on sizes and shapes of tail fins.

As for the windmill design, optimum blade sizes are summarized in Fig. 6, showing an optimum of around 40 $\mathrm{mm}$. In contrast, blade number (from 4 to 16 ) is relatively indifferent among students results (graph is omitted).

These graphs indicate that, the data collection of scattered experimental conditions was also effective to find out optimum conditions of the products, if the member took data quantitively and summarized the data statistically. It is possible that this style of approach is rather matched to improve working conditions of many products since many "real" products essentially contain complexity in themselves.

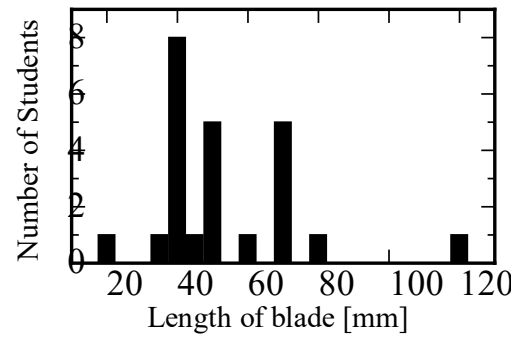

Fig. 6 Optimum blade length of windmills.

\section{Summary}

Optimum sizes and shapes of tail fins and windmills for mini generator kit were determined by quantitative data collection by students' (11-15 in age) homework. The result shows that several specific tendencies when the data summarized statistically, although the data collected with different places with different conditions.

\section{Acknowledgements}

This program was fully supported by JST fund of Junior Doctor Educational program.

\section{References}

1. R. Khunyakari, S. Mehrotra, S. Chunawala, C. Natarajan, Design and technology productions among middle school students: an Indian experience, Int. J. Technol. Des. Educ. 17, 2007, pp. 5-22.

2. Y. Kawamura, K. Honda, O. Izutsu, Y. Matsumoto, Development of educational materials savonius type windmill aerogenerator using handmade generator, J. Jpn. Inst. Energy 92(9), 2013, pp. 913-918.

3. R. Hikiji and Y. Fujimoto, Study on improving experiment-teaching materials of wind power generator for junior high school students to get interested in natural energy, J. JSEE 65(5), 2017, pp. 48-53.

4. J. Concannon and P. L. Brown, Windmills by design: Purposful curriculum design to meet next generation science standards in a 9-12 physics classroom, Science Activities 54(1), 2017, pp. 1-7.

5. Y. Ohnishi, S. Mori, T. Deyama, J. Ono, H. Muko, Development of teaching materials featuring wind power generator for $6^{\text {th }}$ elementary science class based on the relationship with technology class of junior high school, Ehime Univ. Edu. Faculty Kenkyu Kiyo 61, 2014, pp. 105110. 